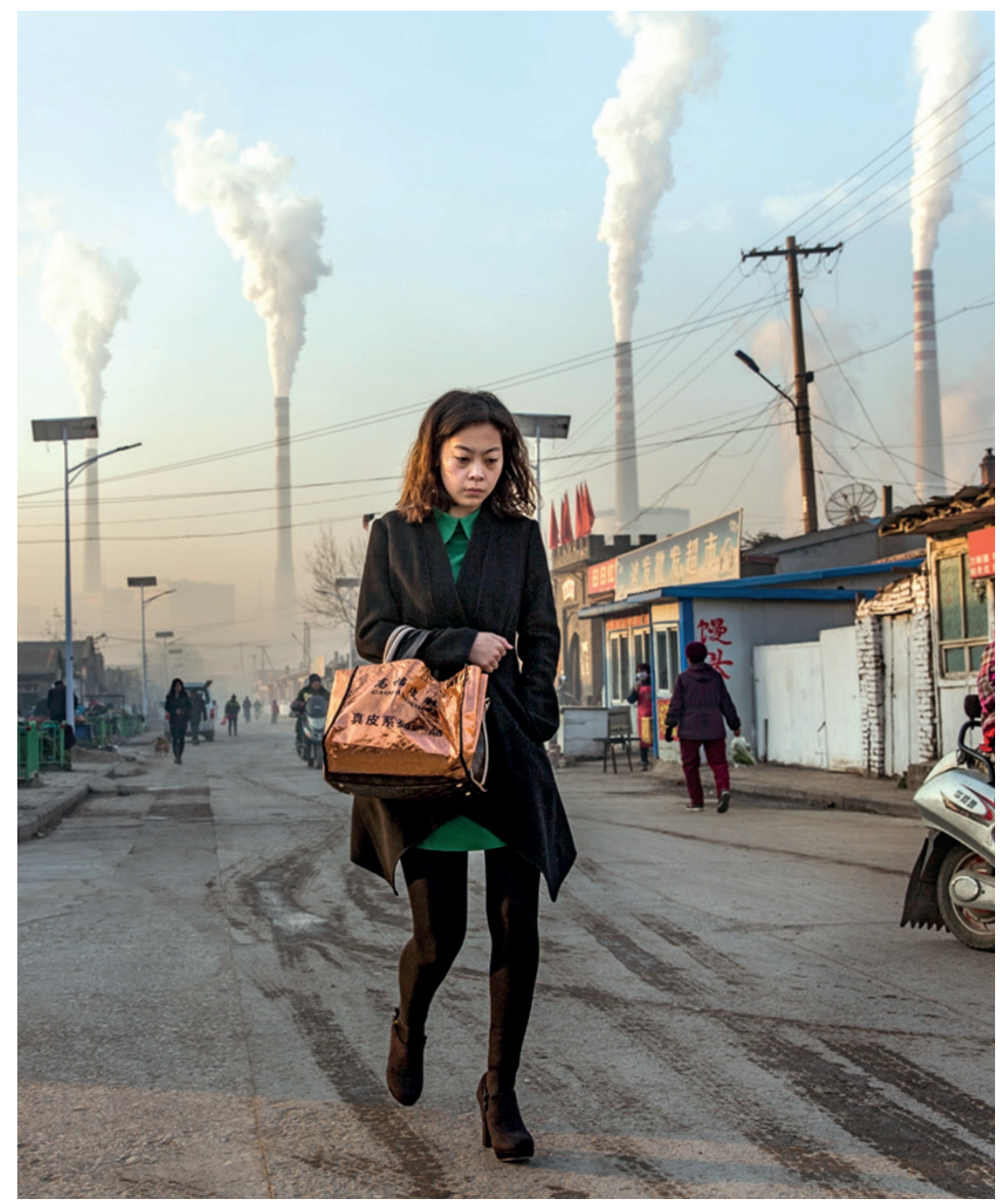

Air pollution is known to be linked with lung cancer, but it is uncertain how many people are in danger.

ENVIRONMENT

\title{
Breathing trouble
}

\section{Large-scale studies are confirming suspicions that air pollution significantly increases the risk of lung cancer.}

\section{BY TRACI WATSON}

$\mathrm{T}$ There are many things people can do to lower their risk of developing lung cancer. They can choose not to smoke, they can stay away from smoky places and they can avoid breathing dirty air. Air pollution is often particularly high in cities, which are home to more than half the global population. With every breath, people living in polluted areas may inhale the same carcinogens as a smoker, including tiny contaminants called particulates.
- microscopic droplets that are made from dust, the byproducts of power plants and other chemical components - as carcinogenic, after reviewing numerous studies linking it to lung cancer (see 'Something in the air').

With the link between air pollution and lung cancer now solid, researchers are embarking on studies to put numbers to the worldwide impact. Initial results indicate a much higher share of lung cancer linked to air pollution than previously thought - one analysis ${ }^{4}$ suggests that in some countries, one form of particulate pollution was a factor in $10-20 \%$ of lung-cancer deaths. "Some estimates are larger than ours, some smaller," says epidemiologist Aaron Cohen of the Health Effects Institute in Boston, Massachusetts, who is one of the study's authors. "But all of them are large enough to be of concern for public health."

\section{THE MURKY METROPOLIS}

The epidemiological research needed to show an association between lung cancer and air pollution in humans is a monumental challenge. Most cases of lung cancer are associated with smoking, which makes it difficult for researchers to disentangle tobacco use from other potential causes. Lung cancer also takes time to develop, so investigators must follow a cohort of people for decades before a sufficient number of non-smokers will be diagnosed with the disease.

And air pollution in general is tricky to study. It is a complicated cocktail of chemicals that waft from sources from factories to lawnmowers. That complexity has frustrated researchers trying to discover what makes air pollution so carcinogenic. A number of studies show that fine particulate matter particles less than 2.5 micrometres in diameter - is a key player. Placing blame on air pollution is also tricky because air quality differs between countries, cities and even neighbourhoods. Many early studies in the United States relied on skimpy networks of airquality monitors and could not capture that variability. "It's a very hard area to study," says environmental health scientist Michael Jerrett of the University of California, Berkeley. "I tell my graduate students they should never study cancer in the environment until they've gotten tenure" and are no longer under such intense pressure to publish rapidly.

But researchers are learning to navigate some of the obstacles. An innovative technique called land-use regression uses monitors to measure air quality at dozens or even hundreds of locations. The data are then used to build an air-quality model that takes factors such as road length and topography into account to predict pollution levels at the address of every person in an epidemiological study - a vast improvement over the old days when researchers assumed everyone in a city was exposed to the same concentrations 
of airborne contaminants. Land-use regression and related techniques "have definitely improved the evidence base a lot", Jerrett says.

These methods were deployed on a grand scale in a 2013 study across nine European countries ${ }^{1}$. The authors estimated exposure to pollution at the addresses of more than 300,000 people, including smokers and non-smokers. They found that a rise by 10 micrograms per cubic millimetre in fine particulate levels - roughly the difference between average pollution levels in a cleaner European city (Oslo) and a dirtier one (Athens) - increases the risk of lung cancer by $40 \%$. The study, one of the largest epidemiological analyses of the link, confirmed previous results in US populations ${ }^{5}$. The results carried substantial weight with the IARC working group that evaluated the evidence linking air pollution with lung cancer, says working-group member Francine Laden, an epidemiologist at the Harvard School of Public Health in Boston, Massachusetts.

\section{NON-SMOKERS BEWARE}

Just over a decade ago, Jonathan Samet, an epidemiologist at the University of Southern California in Los Angeles and the chair of the IARC working group, co-authored a review calling the epidemiological evidence for a link between air pollution and lung cancer "equivocal". But that uncertainty is dissipating rapidly. Scientists have conducted "studies that are really quite different kinds of designs, quite different types of populations, different exposure assessments, and we're still seeing these elevated risks", says Michael Brauer, an environmental health scientist at the University of British Columbia in Vancouver, Canada, who was also a member of the IARC working group. "You can't say this is an erroneous result that's driven by this design feature in this type of study."

To reassure themselves that air pollution is a risk factor for lung cancer, researchers needed data that ruled out exposure to cigagarette smoke. Exactly such a study was published in 2011. The study examined nearly 189,000 people in the United States who had never smoked and took into account their exposure to second-hand smoke. Between 1982 and 2008, 1,100 of those people died of lung cancer, translating to an increased lung cancer-death risk of up to $27 \%$ for every $10 \mu \mathrm{g} / \mathrm{m}^{3}$ rise in fine particulates ${ }^{2}$.

Michael Thun, a co-author of the study and an epidemiologist at the American Cancer Society who is now retired and based in Atlanta, Georgia, confesses that before he collaborated on the study, he had doubts that low levels of air pollution in the United States were sufficient to cause lung cancer. But, he says, "the results in never-smokers substantially strengthened my confidence that the relationship was causal", and he is now convinced.

\section{SOMETHING IN THE AIR}

Global lung-cancer death rates in 2010 attributable to particulate matter, microscopic airborne droplets or particles that can be traced to sources including power-plant chimneys and dusty fields. The link between lung cancer and fine particulate matter - up to 2.5 micrometres in diameter — is especially strong.

12

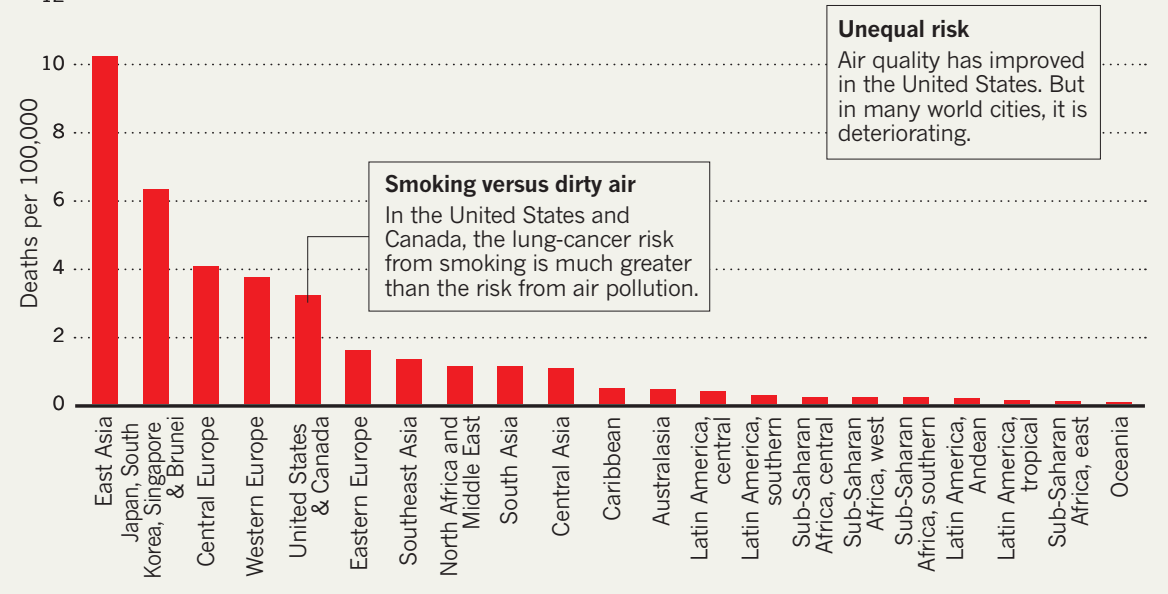

Thun is not the only person to change his mind. Samet now says that the epidemiology shows an association between air pollution and lung cancer, adding that results from studies of large populations are bolstered by laboratory findings and by research on people who breathe in high levels of air pollution at work. The link is now widely accepted by researchers in the field, Brauer says.

\section{NO LOWER LIMIT}

Researchers have yet to find a safe level of air pollution. The European study found a higher risk of lung cancer even at fine-particulate levels lower than the European Union's limit of $25 \mu \mathrm{g} / \mathrm{m}^{3}$, and the authors found no evidence of a threshold - that is, no level of air pollution below which there is no increased risk of developing the disease. The analysis also found a linear relationship between incidence of lung cancer and air pollution levels, at least at concentrations common in developed countries.

Now that a link between air pollution and lung cancer has been established, researchers are building sophisticated models to determine the number of people around the world who develop lung cancer from exposure to it. However, because most research has been focused in areas with low levels of pollution, data investigating the risk posed by outdoor particulate levels of more than $30-40 \mu \mathrm{g} / \mathrm{m}^{3}$ are scant. A team working on one study ${ }^{5}$ sought to estimate the danger of breathing very high levels of particulates by using inhaled tobacco smoke as a proxy. Like the European study, their analysis showed a nearly linear relationship between lungcancer risk and exposure to fine particulates, with no evidence of a threshold.

Another study ${ }^{6}$ combined data indicating the risk posed by smoking and inhaling second-hand smoke as a way to assign risk numbers to countries, such as China, that have very high particulate levels. The results indicate that in many countries, fine particulates played a part in $10-20 \%$ of lung-cancer deaths in 2010. Based in part on the methods used in the paper, the Institute for Health Metrics and Evaluation, a research centre at the University of Washington in Seattle, found that in 2010, outdoor particulate pol-

lution contributed at "The results least in part to 223,000 in neversmokers strengthened lung-cancer deaths worldwide ${ }^{7}$.

The estimates pubmy confidence that the relationship was causal." lished so far come with big uncertainties. No one really knows the size of the risk at the extremely high levels of pollution seen in cities such as Beijing, because most results to date have come from studies in developed countries. To produce firmer numbers, researchers need more data from countries with much more pollution.

It took a long time for scientists to establish the link between air pollution and lung cancer, but it may take a lot longer for that knowledge to save large numbers of lives.

Traci Watson is a freelance science writer in Washington DC.

1. Raaschou-Nielsen, O. et al. Lancet Oncol. 14, 813-822 (2013).

2. Turner, M. C. et al. Am. J. Resp. Crit. Care Med. $\mathbf{1 8 4}$ 1374-1381 (2011)

3. Loomis, D. et al. Lancet Oncol. 14, 1262-1263 (2013).

4. Pope, C. A. III. et al. Environ. Health Perspect. 119, 1616-1621 (2011)

5. Pope, C. A. III. et al. J. Am. Med. Assoc. 287, 1132-1141 (2002)

6. Burnett, R. T. et al. Environ. Health Perspect. 122, 397-403 (2014).

7. Inst. Health Metrics \& Evaluation, Global Burden of Disease Cause Patterns 2010, available at: go.nature.com/brc4nw. 